\title{
Gas phase diagnostics of protoplanetary disc extension (Research Note)
}

\author{
B. Ercolano ${ }^{1}$, J. J. Drake ${ }^{2}$, and C. J. Clarke ${ }^{1}$ \\ ${ }^{1}$ Institute of Astronomy, University of Cambridge, Madingley Road, Cambridge, CB3 OHA, UK \\ e-mail: [be;cclarke]@ast.cam.ac.uk \\ 2 Smithsonian Astrophysical Observatory, MS-3, 60 Garden Street, Cambridge MA 02138, USA \\ e-mail: jdrake@cfa.harvard.edu
}

Received 10 August 2008 / Accepted 28 October 2008

\begin{abstract}
Aims. We investigate the potential of using ratios of fine structure and near-infrared forbidden-line transitions of atomic carbon to diagnose protoplanetary disc extension.

Methods. Using results from 2D photoionisation and radiative transfer modelling of a realistic protoplanetary disc structure irradiated by X-rays from a T Tauri star, we obtain theoretical emission maps from which we construct radial distributions of the strongest emission lines produced in the disc.

Results. We show that ratios of fine structure to near-infrared forbidden-line emission of atomic carbon are especially promising for constraining the minimum size of gaseous protoplanetary discs. While theoretically viable, the method presents a number of observational difficulties that are also discussed here.
\end{abstract}

Key words. accretion, accretion disks - stars: planetary systems: protoplanetary disks - line: formation

\section{Introduction}

Characterising the gas distribution of protoplanetary discs is fundamental to many aspects of star and planet formation, as discs provide the reservoir of material for nascent stellar-planetary systems. In particular, the outer radius is a key parameter for understanding a disc's viscous evolution and its ability to form planets. Indeed, it is the viscous timescale at the outer edge of a disc that controls the rate at which the flow of gas and dust into the inner disc declines (e.g. Hartmann et al. 1998), making it relevant to planet formation at small radii.

The sizes of protoplanetary discs have been determined from direct imaging only in a few cases, e.g., in the optical for sources in the Orion nebula cluster (ONC), where the disc silhouettes can clearly be distinguished (e.g. O’Dell 1993; Bally et al. 1998) and in the millimetre and submillimetre for some nearby objects (e.g., Kitamura et al. 2002; Andrews \& Williams 2008; Wilner et al. 2003; Lommen et al. 2007; Testi \& Leurini 2008, and references therein). Observations show evidence that protoplanetary discs are photoevaporated to smaller sizes by the intense UV flux in cluster environments. Rodmann (2002) analysed the silhouette discs in the ONC and found that $80 \%$ of systems hosting proplyds ${ }^{1}$ contained discs unresolved by the Hubble Space Telescope, and hence smaller than 50 AU. This contrasts with the submillimetre observations of Kitamura et al. (2002) and Andrews \& Williams (2008) in the Taurus-Auriga and Ophiuchus-Scorpius star forming regions, implying a strong environmental dependence.

Models of sub-solar and solar-mass systems (e.g. Fatuzzo \& Adams 2008; Clarke 2007; Adams et al. 2004;

\footnotetext{
1 Identified as compact ionised gas around low mass stars. These systems often show offset cometary-shaped ionisation fronts indicating photoevaporation.
}

Hollenbach et al. 2000) predict that in a cluster environment protoplanetary discs are photoevaporated from the outside in by a strong EUV and FUV radiation field. This has obvious implications for planet formation, which is generally considered to be compromised when the disc evaporation time becomes less than $\sim 10 \mathrm{Myr}$ at a disc radius of $\sim 30$ AU. Fatuzzo \& Adams (2008) conclude that for the distribution of cluster size in the solar neighbourhood, $25 \%$ of the disc population lose their planet forming potential due to FUV radiation and $7 \%$ due to EUV radiation. The effects of photoevaporation on planet formation are predicted to be even more dramatic for lower mass systems (e.g. Laughlin et al. 2004). However, such models have yet to be verified by observations. Comparison of gaseous disc sizes and ages in a variety of environments would help constrain the efficiency of the external photoevaporation process.

For all but the closest systems, however, direct imaging is not possible and disc sizes can only be inferred using indirect techniques. A commonly applied indirect method to determine disc sizes relies on modelling of observations of dust continuum emission. However, the interpretation of the continuum spectral energy distribution (SED) relies on dust radiative transfer modelling and is complicated by a number of known degeneracies between different model parameters. For example, the radial distance of the emitting dust (i.e. the size of the dust disc) is degenerate with respect to the temperature of the irradiating source (stellar, interstellar/cluster radiation fields), with respect to the assumed grain size (which is highly uncertain in discs due to grain growth), and (less dramatically) to the disc chemical state. Further, the temperature of the grains is sensitive to the geometry of the disc (flaring) and to dust settling. These aspects are poorly constrained and lead to significant model-dependence in the SED interpretation (e.g. Alcalá et al. 2008). A further problem with the determination of disc sizes from dust SED 
modelling is that cold dust at large radii only has a very small effect on the emergent SED and may remain undetected. Finally, the estimates of disc size will also depend on whether the disc models used assume a smooth dust distribution or a clumpy one (e.g., Ercolano et al. 2007).

Regardless of the accuracy of the disc size calculations from SED dust modelling, one should also consider circumstances in which the extension of the dust disc is not a good tracer of the real gaseous disc extension. Throop \& Bally (2005), for example, predict a scenario where the gas and the small grains in the outer discs are photoevaporated, while larger grains grow and settle. This would lead an SED analysis to infer larger outer radii than the real gaseous disc extension. The opposite scenario has also been suggested based on recent observations of the extent of dust continuum emission whereby the outer disc appears $d e-$ pleted in dust, perhaps through inward migration (e.g. Isella et al. 2007), while Hughes et al. (2008) show that employing different disc models can resolve the apparent discrepancy. The ongoing debate in the literature underlines the difficulty in obtaining accurate measures of disc size from current observations.

While CO ground-based observations have been employed to obtain estimates of disc sizes (e.g. Dent et al. 2005), gasphase atomic line diagnostics have so far yet to be used to sample gaseous protoplanetary disc sizes. In fact observations of emission lines from gaseous discs have only recently become possible in the IR from space with Spitzer (e.g Lahuis et al. 2007; Espaillat et al. 2007; Pascucci et al. 2007) and from the ground with SMA (Qi et al. 2006) and MICHELLE at Gemini North (Herczeg et al. 2007). Future instrumentation (e.g. Herschel, ALMA, CARMA, JWST) hold the promise for better spectral coverage, resolution and signal to noise (e.g. van Disheock \& Jorgensen 2008). Theoretical identification of viable gas phase diagnostics in support of future observing campaigns is therefore timely. In this paper we suggest that fine structure to near infrared forbidden-line emission ratios of atomic carbon might provide a useful measure of the minimum size of a gaseous T Tauri disc.

\section{Atomic carbon diagnostics of disc extension}

The ratio of fine structure (FS) to near-infrared (NIR) lines of neutral carbon increases as the disc radius gets larger. The main reason for this is the exponential dependence on temperature of these collisionally excited lines that dominates the emitted flux of the NIR lines. The NIR lines are approximately $15000 \mathrm{~K}$ (C I $9826 \AA$ and $9852 \AA$ ) and 31000 K (C I $8729 \AA$ ) above ground, which makes their emissivity negligibly small at the cool temperatures of the outer disc. FS lines, on the other hand, are only $20-40 \mathrm{~K}$ above the ground and therefore they are not very temperature-sensitive.

A comparison of C I FS and NIR lines of different excitation temperatures can therefore, at least in principle, provide a diagnostic for the extension of disc gas. The ratio of these lines is to be preferred to using the absolute flux of C I FS as an indicator as the latter requires an assumption of carbon depletion onto grains, which may be different in different systems. This problem would indeed affect the estimates of disc extension provided by $\mathrm{CO}$ measurements.

We have used the thermal and ionisation structure for a typical T Tauri disc modeled by Ercolano et al. (2008a, Paper I) using the MOCASSIN code (Ercolano et al. 2003, 2005, 2008b) to calculate spatially-resolved emissivities of transitions of neutral carbon. We assume that the direct radiative input to the gas is from X-ray irradiation; at greater depths in the disc, gas-grain
Table 1. Absolute luminosities of C I fine structure and near-infrared lines.

\begin{tabular}{cc}
\hline \hline Wavelength & $\begin{array}{c}\text { Luminosity } \\
{\left[10^{-7} L_{\odot}\right]}\end{array}$ \\
\hline $8729 \AA$ & 4.6 \\
$9826 \AA$ & 3.2 \\
$9852 \AA$ & 9.5 \\
$370 \mu \mathrm{m}$ & 55 \\
$609 \mu \mathrm{m}$ & 11 \\
\hline
\end{tabular}

collisons are important. This latter thus incorporates one aspect also of heating by FUV radiation, since we adopt the grain temperature distribution computed by d'Alessio et al. (2001), which includes the effects of irradiation by the star's optical/FUV spectrum. We do not include the other main aspects of FUV heating, i.e. FUV photoelectric heating, $H_{2}$ vibrational pumping, nor cooling via CO rovibrational lines. FUV photoelectric heating, in particular, may be an important thermal channel, and its omission may cause an error in the gas temperatures derived. This effect will be included in future models.

While we refer to Paper I for a detailed description of the model, it is important to give here at least a brief overview of the gas thermal balance. In the region of the disc producing the lines discussed here (i.e. at relatively low column densities), the main heat input is from X-ray photoionisation, while cooling occurs via collisionally excited lines of metals and $\operatorname{Ly} \alpha$, free-free and free-bound continua and dust-gas collisions. Figure 5 of Paper I shows the relative heating and cooling rates as a function of vertical column at a radial distance of $1 \mathrm{AU}$ for the model in use.

We used a fixed density distribution for the the disc, which was calculated by d'Alessio et al. $(2001,2003,2005)$ to fit the median SED of T Tauri stars in Taurus. We refer the reader to D'Alessio et al. (2001) for a detailed description of the model ingredients and calculation. In brief, the input parameters for this model include a central star of $0.7 M_{\odot}, 2.5 R_{\odot}$ irradiating the disc with an effective temperature of $4000 \mathrm{~K}$. Additional disc parameters consist of a mass accretion rate of $1 . e-8 M_{\odot} \mathrm{yr}^{-1}$ and a viscosity parameter $\alpha=0.01$. The total mass of the disc is $0.027 M_{\odot}$

We have recalculated the emitted flux of the FS lines adding excitation by impact with atomic hydrogen, which is important in the outer disc regions and was not treated in Paper I. Furthermore, the line luminosities reported in Table 1 of Paper I were obtained integrating the disc out to a radius of $190 \mathrm{AU}$. We report here (Table 1) the revised C I FS luminosities, which include collisions with atomic $\mathrm{H}$ and are integrated out to a disc radius of $500 \mathrm{AU}$. When integrated to a radius of $190 \mathrm{AU}$ our revised C I FS line luminosities are $1.8 \times 10^{-7} L_{\odot}$ and $1.1 \times 10^{-6} L_{\odot}$ for C I $609 \mu \mathrm{m}$ and C I $370 \mu \mathrm{m}$, respectively (a factor of 1.4 and 2.8 larger than reported in Paper I). The absolute luminosities of the FS lines reported in Table 1 are in agreement with Hollenbach et al. (1991) who predicted the ubiquitous presence of C I emission from cloud surfaces at the $10^{-5} \mathrm{erg} \mathrm{cm}^{-2} \mathrm{~s}^{-1}$ level, which corresponds to a luminosity of $\sim 2 \times 10^{-6} \mathrm{~L}_{\odot}$ for a $500 \mathrm{AU}$ disc.

Excitation by impact with atomic $\mathrm{H}$ may also contribute to the emissivities of the NIR lines in a similar fashion as for the FS lines discussed above. To our knowledge, the relevant excitation coefficients are not available in the literature and we are therefore unable to provide a robust estimate for the magnitude of this effect. The C I NIR line luminosities reported in Table 1 are thus likely to be underestimated by a factor of order unity. 

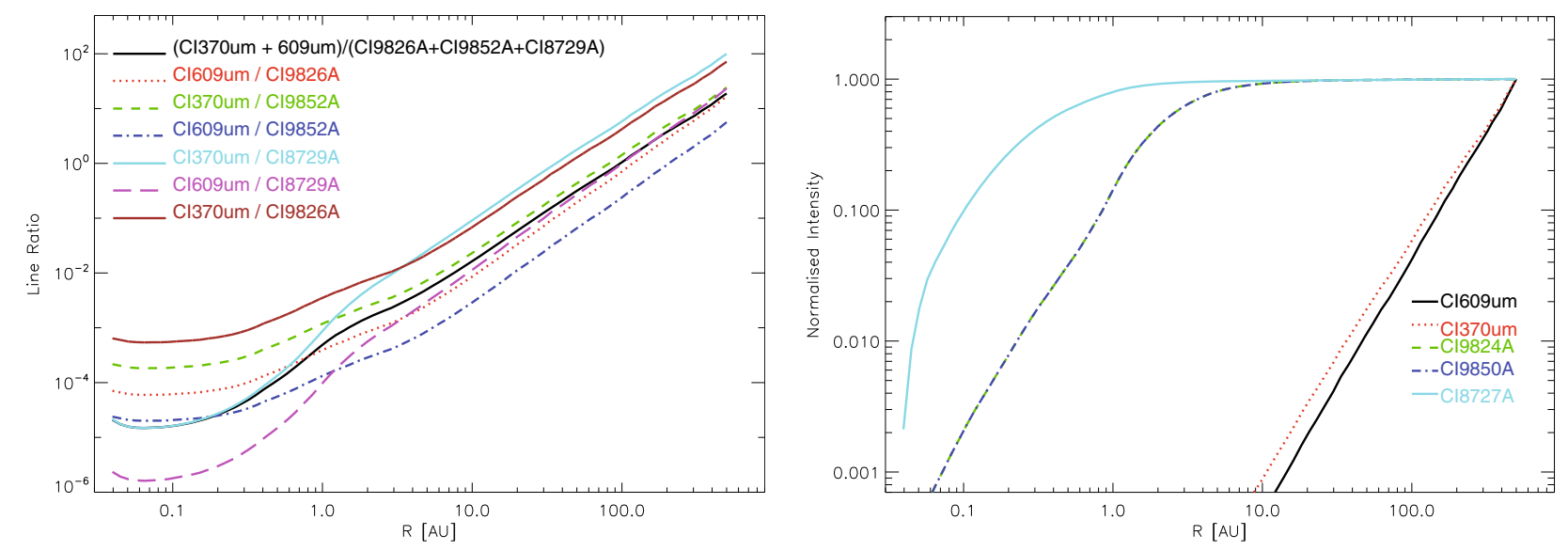

Fig. 1. Left: C I fine structure to NIR collisionally excited line ratios integrated over the volume of the disc as a function of disc outer radius. Right: normalised line fluxes integrated over the volume of the disc as a function of disc size. Note that [C I] $9824.13 \AA$ and $9850.26 \AA$ have a fixed theoretical ratio of 0.34 .

From our theoretical emission maps, we constructed radial distributions of the strongest emission lines produced in the disc. Figure 1 (right) shows the normalised line fluxes integrated over the volume of the disc as a function of disc size. Figure 1 (left) shows the C I FS to NIR line ratios integrated over the volume of the disc as a function of disc size (i.e. integration radius). In our models, values approaching unity indicate a disc size $\gtrsim 100 \mathrm{AU}$. The FS/NIR line ratios for disc sizes of $30 \mathrm{AU}$ are at least a factor of ten smaller than those with sizes of $100 \mathrm{AU}$ or larger. An observational census of discs in cluster environments using these line ratios would provide a direct observational test of disc photoevaporation predictions such as those of Fatuzzo \& Adams (2008).

At small radii $(\$ 30 \mathrm{AU}), \mathrm{X}$-rays produced by the YSO are most likely the dominant ionising radiation source (e.g. Meijerink et al. 2008; Glassgold et al. 2004). Although significant amounts of EUV radiation are emitted by YSOs (Alexander et al. 2005; Herczeg \& Hillenbrand 2008), which may play an important role in photoevaporating the disc (Shang et al. 2002), it is likely that such radiation will only be able to penetrate to the disc surface at late times, when partially ionised winds, launched close to the star, have become optically thin to Lyman continuum photons. We thus assume that over the majority of a star's disc bearing lifetime, the EUV field that can reach the disc at larger radii and depths is significantly attenuated (Glassgold et al. 2007). External UV radiation fields, however, may become important at larger radii and may affect low-excitation lines (see discussion in Sect. 3). The models of Ercolano et al. (2008a) did not include external UV and FUV radiation fields, and therefore one should further note this as a potential cause of errors in the calculated neutral carbon column.

It should also be mentioned that, as well as collisional excitation of the ground-state $\mathrm{C}\left({ }^{3} \mathrm{P}\right), \mathrm{CO}$ dissociation, dissociative recombination of $\mathrm{CO}^{+}$and collisional dissociation of $\mathrm{CO}$ could all be contributors to the formation of of the C I NIR lines. In the case of protoplanetary discs, however, we expect collisional excitation of $\mathrm{C}\left({ }^{3} \mathrm{P}\right)$ to be by far the dominant mechanism for the emission of the NIR lines, since they are emitted preferentially in a layer where $\mathrm{CO}$ has already completely dissociated and carbon is mostly atomic or singly ionised. At 1 AU Glassgold et al. (2004) calculate that the transition from atomic carbon to CO occurs at a vertical column density of approximately $10^{21} \mathrm{~cm}^{-2}$, and, as shown in Fig. 2, the bulk of the emitted flux comes from warm gas at columns of $\sim 10^{20}$. The electron temperature in this region, shown by asterisks in Fig. 2, is approximately $4000 \mathrm{~K}$ and the electron densities in the NIR emitting regions are $\sim 10^{4}-10^{5} \mathrm{~cm}^{-3}$. We also note that the lines 9826 and 9852 are $14668 \mathrm{~K}$ above ground and the $8729 \mathrm{~A}$ is $31154 \mathrm{~K}$ above ground, so the latter should be preferentially produced in the hotter gas closer to the surface, however higher in the disc atmosphere carbon becomes ionised causing the emissivity to drop.

\section{Discussion}

The gas phase diagnostics we have identified and plotted in Fig. 1 offer a promising new avenue for the study of gaseous $\mathrm{T}$ Tauri discs, but a number of observational considerations should be taken into account and the detection and measurement of the necessary emission lines still presents a challenge for current space-based and ground-based instrumentation.

The main problem facing the ground state forbidden transitions near $1 \mu \mathrm{m}$ is their appearance against the background photospheric continuum of the parent T Tauri star. We have estimated the expected line-to-continuum contrast using the emergent photospheric flux predicted by an ATLAS12 solar metallicity model atmosphere (Castelli \& Kurucz 2004; Kurucz 2005), corresponding to the stellar parameters adopted for the disc models of Ercolano et al. (2008a): effective temperature $T_{\text {eff }}=$ $4000 \mathrm{~K}$, surface gravity $\log g=3.5$, and radius $R=2.5 R_{\odot}$. This model yields equivalent widths for the [C I] lines in emission of 1.7, 5.0 and $2.2 \mathrm{~m} \AA$ for $9824.13 \AA$, $9850.26 \AA$ and $8727.13 \AA$, respectively. While such equivalent widths can in principle be observed, these [C I] lines will also be present in absorption in $\mathrm{T}$ Tauri photospheric spectra.

These [C I] lines were first investigated in detail in the solar spectrum by Lambert \& Swings (1967), who found the cleanest line to be [C I] $8727 \AA$, with an equivalent width of $6.5 \mathrm{~m} \AA$. This line lies in the far wing of a much stronger Si I line, but is otherwise only blended with a very weak Fe I line whose contribution to the $8727.13 \AA$ feature is estimated to be a few percent at most (Lambert \& Swings 1967). Gustafsson et al. (1999) estimated an upper limit of $15 \%$ for the coolest $\left(T_{\text {eff }} \sim 5700 \mathrm{~K}\right)$, most Fe-rich solar-type star of their study of $\mathrm{C}$ abundances in the Galactic disc. Instead, the $9824.13 \AA$ and $9850.26 \AA$ features were not detected and, while they have been observed in emission in spectra of planetary nebulae, $\mathrm{H}$ II and photodissociation regions (e.g. Liu et al. 1995), they have received scant attention in the stellar literature in the intervening years. 


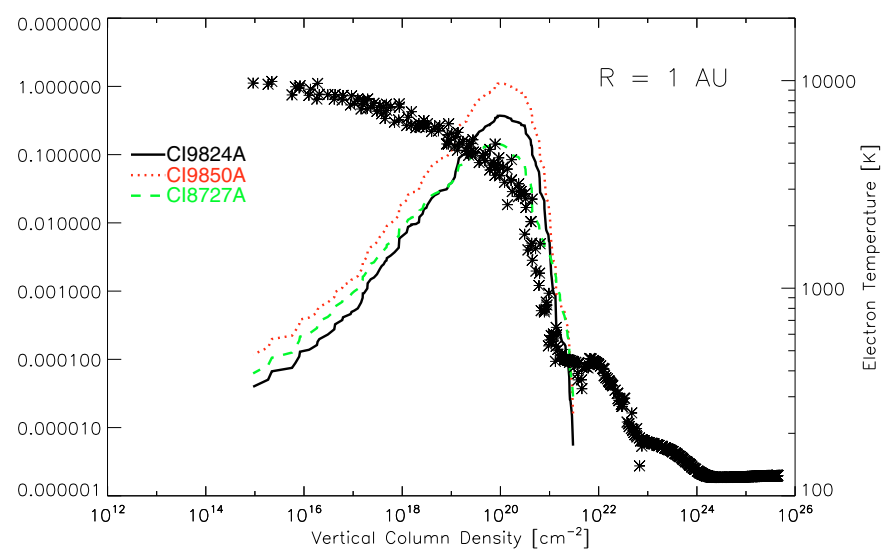

Fig. 2. Normalised emissivities of the near-infrared C I forbidden-lines (solid lines) plotted as a function of vertical column density at a radial distance of 1 AU. Electron temperatures are also plotted here with black asterisks.

One exception is the study of $\mathrm{R}$ Coronae Borealis stars by Pandey et al. (2004), who report measurements of [C I] $9850.26 \AA$ obtained close to a neighbouring line of Fe II, but note that [C I] $9824.13 \AA$ is irretrievably blended. Indeed, the latter falls between telluric water vapour features (which are not necessarily problematic provided accurate correction can be achieved) but also between, and on the far wings of, lines of $\mathrm{CN}$. These $\mathrm{CN}$ lines are very weak in the solar spectrum, with equivalent widths of only a few $m \AA$, but are likely to become stronger in the cooler atmospheres of $\mathrm{T}$ Tauri stars. We note that a very weak $\sim 1 \mathrm{~m} \AA$ feature in the solar spectrum blueward of [C I] $9850.26 \AA$ near $9850.1 \AA$, and possibly the same feature identified as due to Fe II by Pandey et al. (2004), is also coincident with two lines of $\mathrm{Fe} \mathrm{I}$ in the line database of Kurucz (Kurucz, personal communication). A telluric water vapour line lies to the red of the predicted [C I] 9850.26 $\AA$ location at $9850.5 \AA$, but no other conspicuous absorption features are present.

In summary, the lack of any direct coincidence between the predicted location of [C I] 9824.13, 9850.26 $\AA$ and other strong photospheric lines leads us to conclude that further investigation of these features, as well [C I] $8727.13 \AA$, for possible emission signatures in T Tauri stars is well-motivated. Emission line equivalent widths of $1 \mathrm{~m} \AA$ are clearly challenging in any spectral range when superimposed on a background of possible blending lines. For a resolving power of $\lambda / \Delta \lambda \sim 100000$, for example, $1 \mathrm{~m} \AA$ corresponds to a peak flux of order $1 \%$ of the continuum at $9000 \AA$, and requires a signal-to-noise ratio of a few hundred for a firm detection. As we discuss below, the uncertainties inherent in our calculations render our line strength estimates somewhat uncertain: equivalent widths a few times stronger (or weaker) are quite possible. The study of Pandey et al. (2004) using the McDonald $2.7 \mathrm{~m}$ telescope and cross-dispersed echelle observed at $\lambda / \Delta \lambda=120000$ and reached to $J=9$ with signal-to-noise ratio of $>100$. Relatively nearby $\mathrm{T}$ Tauri stars such as those in the Taurus-Auriga star-forming regions have typical $J$-band magnitudes of order 9-12. For these ranges of near infrared magnitudes, equivalent widths of a few $\mathrm{m} \AA$ are quite accessible to current high resolution spectrographs on larger telescopes able to operate in the near-IR.

With regards to the FS C I lines, it should be mentioned here that both the $370 \mu \mathrm{m}$ and the $609 \mu \mathrm{m}$ lines fall in the wavelength range of ALMA and Herschel, with good prospect of these lines being observed in protoplanetary discs in the future. The importance of these lines in the study of protoplanetary discs was indeed already recognised by Jonkheid et al. (2006) who made predictions for the intensities and line profiles for the three [C I] fine structure lines using their chemical model of the transitional disc around HD 141569A. These authors point out that neutral carbon, which is the dominant form of carbon in large parts of the disc, is also a good tracer of disc mass.

We conclude with a discussion of the modelling uncertainties that may affect our predicted C I line fluxes. Ercolano et al. (2008a) discuss a number of limitations of their models, in particular the fact that the disc density structure was calculated assuming full thermal coupling between dust and gas, which they show to be a poor approximation in the warm disc atmosphere. Furthermore, line ratios may also vary for different X-ray luminosities and may also be sensitive to additional spectral components (for example, the inclusion of a strong FUV field would affect the abundance of neutral carbon, both positively, via the photodissociation of $\mathrm{CO}$, and negatively, through photoionisation of neutral carbon). Finally the C I NIR line intensities are very sensitive to the gas temperature in the dominant emitting zone, and therefore uncertainties in the model-determined thermal structure will affect the NIR/FS ratio. Although forthcoming models will aim at remedying these shortcomings, these important caveats should be taken into account when considering the line diagnostics proposed here, which are not intended to be used in order to provide precise measures of disc sizes. An observational study based on comparison of fine structure to NIR forbidden-lines of atomic carbon should be carried out in a statistical fashion, by comparing the mean ratios measured in multiple objects belonging to different environments, such as dense clusters like the ONC or low density regions like Taurus-Auriga. An interesting comparison would also be between regions of different ages.

The prospect of observational studies based on emission lines produced in gaseous protoplanetary discs will be greatly improved when ALMA, CARMA, Herschel and later JWST come on-line. The work presented here aims at contributing to the construction of a theoretical framework in aid of future observational campaigns.

Acknowledgements. We thank the anonymous referee for a thorough and constructive assessment of our work. J.J.D. was funded by NASA contract NAS839073 to the Chandra X-ray Center during the course of this research. The simulations were partially run on the Cosmos (SGI altix 4700) supercomputer at DAMTP in Cambridge. Cosmos is a UK-CCC facility which is supported by HEFCE and STFC.

\section{References}

Adams, F. C., Hollenbach, D., Laughlin, G., \& Gorti, U. 2004, ApJ, 611, 360 Alcalá, J. M., Spezzi, L., Chapman, N., et al. 2008, ApJ, 676, 427 Alexander, R. D., Clarke, C. J., \& Pringle, J. E. 2005, MNRAS, 358, 283 Andrews, S. M., \& Williams, J. P. 2007, ApJ, 659, 705

Bally, J., Sutherland, R. S., Devine, D., \& Johnstone, D. 1998, AJ, 116, 293 Castelli, F., \& Kurucz, R. L. 2004, [arXiv: astro-ph/0405087] Dent, W. R. F., Greaves, J. S., \& Coulson, I. M. 2005, MNRAS, 359, 663 D’Alessio, P. 2003, Rev. Mex. Astron. Astrofis. Conf. Ser., 18, 14 D'Alessio, P., Calvet, N., \& Hartmann, L. 2001, ApJ, 553, 321

D’Alessio, P., Merín, B., Calvet, N., Hartmann, L., \& Montesinos, B. 2005, Rev. Mex. Astron. Astrofis., 41, 61

Dullemond, C. P., Dominik, C., \& Natta, A. 2001, ApJ, 560, 957

Ercolano, B., Barlow, M. J., Storey, P. J., \& Liu, X.-W. 2003, MNRAS, 340, 1136

Ercolano, B., Barlow, M. J., \& Storey, P. J. 2005, MNRAS, 362, 1038

Ercolano, B., Barlow, M. J., \& Sugerman, B. E. K. 2007, MNRAS, 375, 753

Ercolano, B., Drake, J. J., Raymond, J. C., \& Clarke, C. C. 2008a, ApJ, 688, 398 
Ercolano, B., Young, P. R., Drake, J. J., \& Raymond, J. C. 2008b, ApJS, 175, 534

Espaillat, C., Calvet, N., D’Alessio, P., et al. 2007, ApJ, 664, L111

Fatuzzo, M., \& Adams, F. C. 2008, ApJ, 675, 1361

Glassgold, A. E., Najita, J., \& Igea, J. 2004, ApJ, 615, 972

Glassgold, A. E., Najita, J. R., \& Igea, J. 2007, ApJ, 656, 515

Gustafsson, B., Karlsson, T., Olsson, E., Edvardsson, B., \& Ryde, N. 1999, A\&A, 342, 426

Hartmann, L., Calvet, N., Gullbring, E., \& D’Alessio, P. 1998, ApJ, 495, 385

Herczeg, G. J., \& Hillenbrand, L. A. 2008, ApJ, 681, 594

Herczeg, G. J., Najita, J. R., Hillenbrand, L. A., \& Pascucci, I. 2007, ApJ, 670, 509

Hollenbach, D. J., Takahashi, T., \& Tielens, A. G. G. M. 1991, ApJ, 377, 192

Hollenbach, D. J., Yorke, H. W., \& Johnstone, D. 2000, Protostars and Planets IV, 401

Hughes, A. M., Wilner, D. J., Qi, C., \& Hogerheijde, M. R. 2008, ApJ, 678, 1119

Isella, A., Testi, L., Natta, A., et al. 2007, A\&A, 469, 213

Jonkheid, B., Kamp, I., Augereau, J.-C., \& van Dishoeck, E. F. 2006, A\&A, 453, 163

Kitamura, Y., Momose, M., Yokogawa, S., et al. 2002, ApJ, 581, 357
Kurucz, R. L. 2005, Mem. Soc. Astron. It. Suppl., 8, 14

Lahuis, F., van Dishoeck, E. F., Blake, G. A., et al. 2007, ApJ, 665, 492

Lambert, D. L., \& Swings, J. P. 1967, Sol. Phys., 2, 34

Laughlin, G., Bodenheimer, P., \& Adams, F. C. 2004, ApJ, 612, L73

Liu, X.-W., Barlow, M. J., Danziger, I. J., \& Clegg, R. E. S. 1995, MNRAS, 273, 47

Lommen, D., Wright, C. M., Maddison, S. T., et al. 2007, A\&A, 462, 211

Meijerink, R., Glassgold, A. E., \& Najita, J. R. 2008, ApJ, 676, 518

O'dell, C. R., Wen, Z., \& Hu, X. 1993, ApJ, 410, 696

Pandey, G., Lambert, D. L., Rao, N. K., et al. 2004, MNRAS, 353, 143

Pascucci, I., Hollenbach, D., Najita, J., et al. 2007, ApJ, 663, 383

Qi, C., Wilner, D. J., Calvet, N., et al. 2006, ApJ, 636, L157

Robitaille, T. P., Whitney, B. A., Indebetouw, R., Wood, K., \& Denzmore, P. 2006, ApJS, 167, 256

Rodmann, J. 2002, Diploma Thesis, Univ. Potsdam, Germany

Shang, H., Glassgold, A. E., Shu, F. H., \& Lizano, S. 2002, ApJ, 564, 853

Testi, L., \& Leurini, S. 2008, New Astron. Rev., 52, 105

Throop, H. B., \& Bally, J. 2005, ApJ, 623, L149

van Dishoeck, E. F., \& Jørgensen, J. K. 2008, Ap\&SS, 313, 15

Wilner, D. J., Bourke, T. L., Wright, C. M., et al. 2003, ApJ, 596, 597 